\title{
EVALUATION OF THE ACCURACY OF PANORAMIC RADIOGRAPH IN THE DEFINITION OF MAXILLARY SINUS SEPTA
}

\author{
Samer Kasabah, Radovan Slezák, Antonín Šimůnek, Jiři Krug, Miguel Cevallos Lecaro
}

Charles University in Prague, Faculty of Medicine in Hradec Králové: Department of Dentistry

\begin{abstract}
Summary: The purpose of this study was to determine the accuracy of panoramic radiography in identification of maxillary sinus septa. Out of 68 sinuses were radiographically examined using both panoramic and computerized tomographic radiographs (CT scan). Using CT scan, 24 (35.9\%) out of 68 cases maxillae showed at least one septum, 22 sinuses (32.3 \%) showed one septum, whereas two sinuses (2.9\%) exhibited two septa. Panoramic radiograph led to a false diagnosis regarding the presence or absence of sinus septa in 18 of 68 sinuses $(26.5 \%)$. On the other hand, they gave negative diagnosis of sinus septa in 12 of 24 septa (50\%). There was fully agreement between the two methods (positive septa) only in 12 of 24 septa (50\%). We cannot depend on panoramic radiograph for the detection of sinus septa because it can lead to false or negative results.
\end{abstract}

Key words: Maxillary sinus; Underwood's septa; Panoramic radiography; CT scan

\section{Introduction}

The maxillary sinus septa were first described by Underwood in 1910 and are thus also referred to as Underwood's septa (12). These septa are barriers of cortical bone and may be varied in number, thickness, and length and they even may divide the sinus into two or more cavities that may communicate $(1,6,9)$.

Etiologically, sinus septa constitute partly congenital, partly acquired malformations. Congenital septa, also referred to as "primary septa", can develop in all maxillary sinus regions (e.g., the floor, the anterior wall) and evolve during the growth of the middle part of the face (4). In contrast, Vinter et al. (13) observed that atrophy of the maxillary alveolar proceeds irregularly in different regions and leaves bony 'crests' on the maxillary sinus floor. Therefore, incomplete septa on the sinus floor, also known as 'secondary septa', can be found because of different reasons. They can be a result of tooth loss and to various phases of maxillary sinus pneumatization. This assumption is also supported by the fact that the sinus floor anterior and posterior to the septum often was on different levels (6). Another explanation is that these septa act as a masticatory force carry struts during the dentate phase of life and seem to disappear slowly when teeth have been lost (1). In addition, they can act to strengthen the maxillary sinus structure (5).

For decades, these septa were considered clinically insignificant anatomical variations. However, detailed knowledge of maxillary anatomy has become increasingly important after the introduction of maxillary sinus lift elevation as a good solution to increase the posterior available bone in maxilla that increase the success rate of the dental implant. Such this knowledge allows more exact planning of invasive surgery and helps to avoid complications since the presence of sinus septa can cause difficulties during sinus lift procedure such as they can limit the creation of a window in the lateral sinus wall and elevation of a hinged door or making difficult to prevent perforation of sinus mucosa during elevation it from an alveolar recess containing septa $(3,10)$ (Fig.1).

The aim of this study was to determine whether it is possible to differentiate the sinus septa clearly on panoramic radiographs.

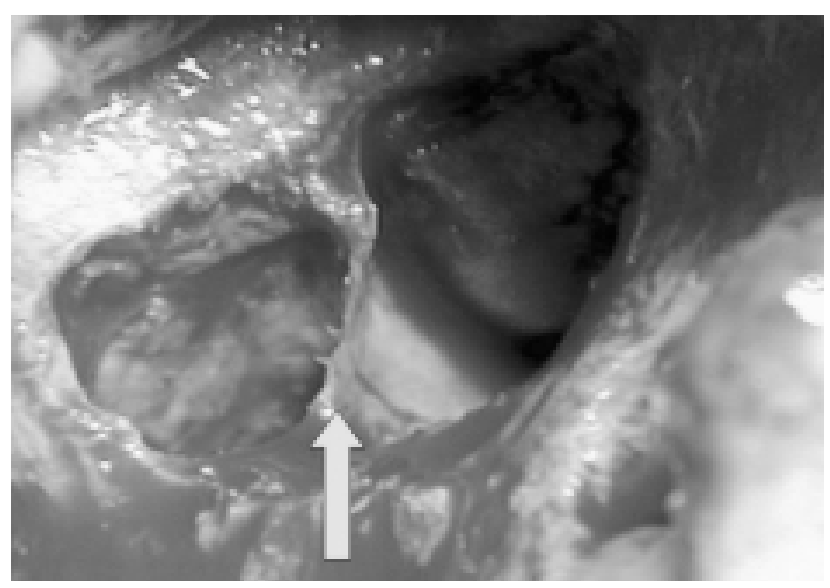

Fig. 1: Intra-operative view of a complete sinus septum that divides the sinus into two cavities (arrow). 


\section{Material and method}

This study was based on 34 pairs of panoramic radiographs and computerized tomographic radiographs (CT scan) [Somatom Plus 4 (Siemens, Erlangen, feed and slice thickness: $1 \mathrm{~mm}$; window width: 2600 ; middle position: 500 HU; 2 s, 500 mAs, image matrix: $512 \times 512$ )] of non-selected adult patients ( 14 females and 20 males) who had been required both panoramic and $\mathrm{CT}$ scan radiographies for preoperative evaluation. The sinus septa were evaluated using mainly an axial plane and the coronal plane of section. The two sets of images were assessed independently by a single dental implantologist (SK) and then the data were confirmed and reviewed by some other authors. The incidence of sinus septa was evaluated using CT scan was compared with the data obtained from panoramic radiography. CT scan was considered as a standard parameter according to the data obtained from panoramic radiographies that were judged. Only those bone lamellae were considered as septa that showed a height of at least 2.5 $\mathrm{mm}$. The term "false septa" indicate the septa that were proposed in panoramic radiograph but were not present in CT scan. We referred to the septa that were not noticed in panoramic but could be observed in CT scan as "negative septa". "Positive septa" defines the septa that were recognized in panoramic and were confirmed by CT scan projections. Mean values were compared using screening test.

\section{Results}

Using CT scan, sinus floor with at least one septum were observed in 24 sinuses (35.9\%) (Fig. 2, 3), 22 sinuses (32.3\%) showed one septum, whereas two sinuses $(2.9 \%)$ exhibited two septa. Panoramic radiographies led to false diagnosed septa in 18 of 68 cases $(26.5 \%)$. On the other hand, they gave negative diagnosis of sinus septa in 12 of 24 septa $(50 \%)$. There were positive septa only in 12 of 24 septa (50\%). Using the CT scan as a standard criterion, the sensitivity of screening test (positivity in panoramic for septa that are absent in CT) was 0.50 which means that there is only 50 percent chance for differentiate sinus septa using panoramic radiograph. The specificity of screening test (negativity in panoramic radiograph for septa that are present in CT scan) was 0.59 that means that there is 59 percent chance to find of negative sinus septa using panoramic radiograph (Tab. 1).

Tab. 1: The results of a screening test applied to the presence and the absence of sinus septa using both the radiographic projections.

\begin{tabular}{|l|c|c|}
\hline \multirow{2}{*}{$\begin{array}{l}\text { Panoramic } \\
\text { radiograph }\end{array}$} & \multicolumn{2}{|c|}{ CT scan } \\
\cline { 2 - 3 } & Septa presence & Septa absence \\
\hline Septa presence & 12 & 18 \\
\hline Septa absence & 12 & 26 \\
\hline
\end{tabular}

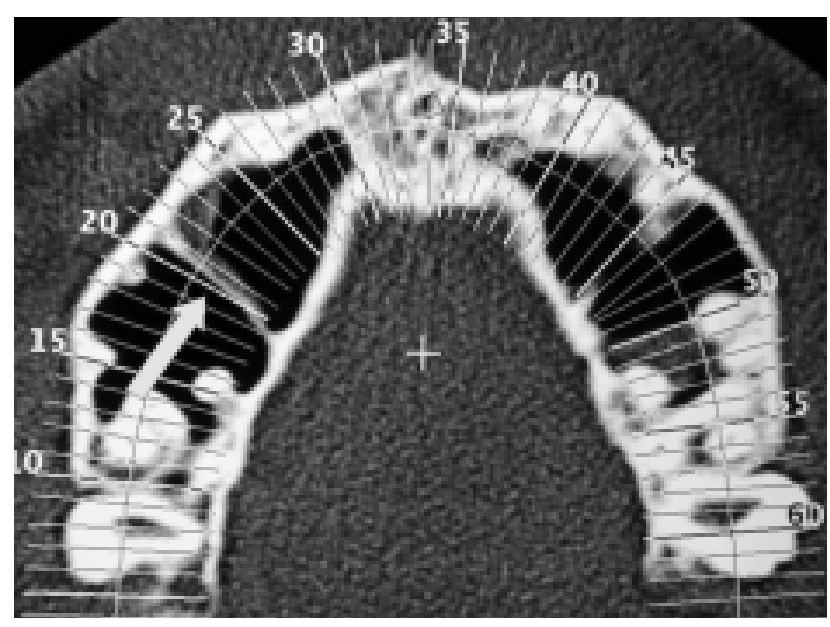

Fig. 2: Axial CT scan showing a complete, thin septum on one side of the sinus (arrow).

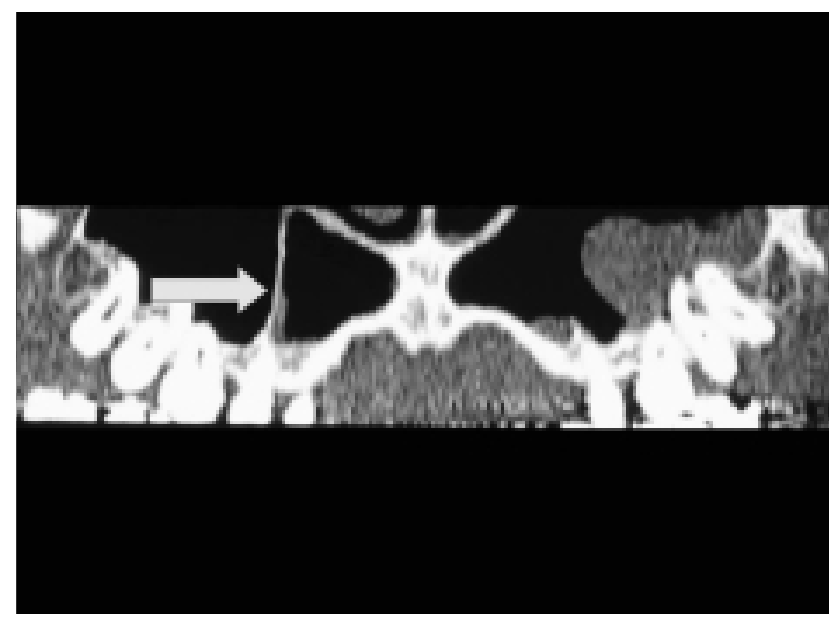

Fig. 3: Sagittal reconstruction corresponding to Figure 1, showing a complete septum of the sinus floor (arrow).

All septa showed frontal or largely frontal orientation (i.e., they were oriented in a buccopalatal plane). Sagittal septa, or septa that followed the arch of the alveolar process, were not observed. It was noticeable that the sinus floor anterior and posterior to the septum often was on different levels.

\section{Discussion}

It is difficult to compare between the panoramic radiographic data with the clinical one in relation to the presence of sinus septa because the septa are not always visible in the surgical site. The panoramic data, of this study, were compared to the CT scan that it the high-resolution imaging of delicate bone structures and that could be considered the method of choice for visualization of sinus septa (8).

According to literature, the incidence of sinus septa varies between $16 \%$ and $58 \%$ (1). Underwood (12) found 30 
septa in 45 skulls (90 maxillary sinuses). Ulm et al (11) found nearly the same incidence $(31.7 \%)$ in anatomical specimens of atrophic maxillae. In clinically preselected population, Jensen and Greer (7) found anatomic variations in 15 out of 26 patients. Betts and Miloro (2) estimated the incidence of maxillary sinus variations to be about $20 \%$. The incidence of radiographically discernible sinus septa in non-preselected patients in this study (35.9\%) is quite similar to that in the above mentioned studies. Nevertheless, the value is high enough to attain significance in clinical routine.

Only bone lamellae were considered as septa when they showed a height of at least $2.5 \mathrm{~mm}$. This criterion was taken from Ulm et al's study published in the International Journal of Oral \& Maxillofacial Implants in 1995 (11). Thus was possible to exclude from the analysis uneven patches of the alveolar recess, which can be observed in any sinus floor.

The great number of the false diagnoses established by the use of panoramic radiograph indicates that it is not suitable enough for thorough evaluation of the sinus septa. The panoramic radiograph often demonstrates the maxillary sinus as multicompartmented, with radiopaque bone septa. This can be interpreted by the fact that the image of the maxillary sinus is found to be transversed by an occasional, or several, radiopaque lines in panoramic radiographs that can gives false septa. These lines can artifact corresponding in its lower half to the posterior surface of the zygomatic process of the maxilla, and in its upper half to the posterior surface of the frontal process of the zygoma. It should be distinguished from the vertical septa, reinforcement webs, or the posterior wall of the sinus. Moreover, the x-ray beam of panoramic radiographies is seldom directed tangentially to these structures that can lead to negative results. In contrast, modern CT scan and subsequent reconstruction allow high-resolution of delicate bone structures, it therefore can be considered the method of choice for visualization of sinus septa. The differences in results between CT and panoramic radiography were large, but probably cannot be attributed to interobserver variation, even though such variation may exist. Axial sections, as have been used in this study, run perpendicular to the orientation of such septa and can be considered the optimal sectional plane for visualization of these bony variations.

\section{Conclusion}

Comparatively to CT scan, panoramic radiograph cannot clearly differentiate the sinus septa from some different other anatomic structures resulting from superimpositioning effects. It can give false negative results.

\section{References}

1. Bergh van den JPA, Bruggenkate ten CM, Disch FJM, Tuinzing DB. Anatomical aspects of sinus floor elevations. Clin Oral Implant Res 2000;11:256-65.

2. Betts NJ, Miloro M. Modification of the sinus lift procedure for septa in the maxillary antrum. Int J Oral Maxillofac Surg 1994;52:332-3

3. Boyne PJ, James RA. Grafting of the maxillary sinus floor with autogenous marrow and bone. Int J Oral Maxillofac Surg 1980;38:613-7.

4. Chanavaz M. Maxillary sinus: anatomy, physiology, surgery, and bone grafting related to implantology-eleven years of surgical experience (1979-1990). J Oral Implantol 1990;16:199-209.

5. Chris M, Bruggenkate T, Bergh van den JPA. Maxillary sinus floor elevation: a valuable pre-prosthetic procedure. Periodontology 2000 1998;17:176-82.

6. Garg AK. Augmentation grafting of the maxillary sinus for placement of Dental Implants: Anatomy, physiology, and procedures. Implant Dent 1999;8:36-46.

7. Jensen OT, Greer R. immediate placement of osseointegrating implant into the maxillary sinus augmented with mineralised cancellous allograft and Gore-Tex: Second-stage surgical and histologic findings. In: Laney WR and Tolman DE (ed): Tissue integration in oral orthopedic and maxillofacial reconstruction. Chicago: Quintessence, IL, 1992:321-33.

8. Mafee MF, Chow JM, Meyers R. Functional endoscopic sinus surgery: Anatomy, CT-Screening, Indications and Complications. Am. J. Radiol 1993;160:735-44.

9. Misch CE. The maxillary sinus lift and sinus graft surgery In: Misch CE. Contemporary in implant dentistry. Mobsy, 1998: Chapter 30.

10. Tidwell JK, Blijdorp PA, Stoelinga PJW, Brouns JB, Hinderks F. Composite graft ing of the maxillary sinus for placement of endosteal implants-a preliminary report of 48 patients. Int J Oral Maxillofac Surg 1992;21:204-9.

11. Ulm CW, Solar P, Krennmair G, Matejka M, Watyek G. Incidence and suggested surgical management of septa in sinus-lift procedures. Int J Oral \& Maxillofac Implants 1995;10(4):462-5.

12. Underwood AS. An inquiry into the anatomy and pathology of the maxillary sinus. J Anat Physiol 1910;44:354.

13. Vinter I, Krmpotic-Nemanic J, Jalsovec D. Verschwindet der Processus alveilaris des Oberkiefers immer nach dem Zahnausfall? Laryngorhinootologie 1993;72: 606-7.

Submitted June 2002.

Accepted September 2002.

Samer Kasabah, DDS,

Charles University in Prague, Faculty of Medicine in Hradec Králové, Department of Dentistry,

Center of Dental Implantology, 50005 Hradec Králové, Czech Republic.

e-mail: skasabah@hotmail.com 